\title{
PTU-055 $九$ A STUDY OF THE PREVALENCE OF GENETIC POLYMORPHISMS IN BILE ACID DIARRHOEA PATIENTS
}

doi:10.1136/gut.2011.239301.183

S Pattni, ${ }^{*}$ S Pathmasrirengan, ${ }^{2}$ P H Dixon, ${ }^{3}$ C Williamson, ${ }^{3}$ J R F Walters ${ }^{1}$ ${ }^{1}$ Gastroenterology, Imperial College London, London, UK; ${ }^{2}$ Imperial Medical School, Imperial College London, London, UK; ${ }^{3}$ Maternal and Fetal Disease, Institute of Reproductive and Developmental Biology, Imperial College London, London, UK

Introduction Bile acid diarrhoea (BAD) is a common cause of chronic diarrhoea and results from high concentrations of bile acids in the colon. It has recently been suggested that impaired production of fibroblast growth factor 19 (FGF19) in the ileum can result in excessive hepatic bile acid synthesis through reduced negative feedback regulation, and this, rather than malabsorption, is the cause of the diarrhoea. The reason for the reduced ileal FGF19 production remains unclear but it has been established that ileal FGF19 synthesis is markedly stimulated by bile acids binding to the farnesoid $\mathrm{X}$ receptor (FXR). In the liver, FGF19 acts through the FGFR4 receptor and its co-receptor Klotho $\beta$. Organic solute transporter $\alpha(\mathrm{OST} \alpha)$ is the ileal basolateral bile acid transporter. The aim of this study was to determine the prevalence of 6 common single nucleotide polymorphisms (SNPs) in four genes involved in bile acid metabolism and FGF19 action, comparing the prevalence in patients with primary $\mathrm{BAD}$, a similar number of controls with diarrhoea, and a larger numbers of healthy controls.

Methods Two common SNPs (rs948992 and rs1789170) in the FGF19 gene were analysed as well as 2 SNPs (M173T, rs61755050 and -1g>t, rs56163822) in the FXR gene. Mutations in FXR are implicated in patients with obstetric cholestasis and have been shown to reduce function and translation efficiency of FXR. A SNP in Klotho $\beta$ (rs17618244) has been associated with colonic transit in IBS-D patients. A further SNP in the OST $\alpha$ gene (rs939885) was also studied.

Genomic DNA was extracted from 92 primary BAD patients diagnosed on the basis of an abnormal ( $<15 \%)$ SeHCAT test and 75 diarrhoea controls. The frequencies of some of these SNPs in our local healthy population has been previous reported. Genotyping of the SNPs was performed using Taqman 5' allelic discrimination assays.

Results Polymorphisms in all the genes studied were detectable in controls and in cases. No significant differences in the genotypes and allele frequencies were found in the Caucasian cohorts in the control and primary BAD groups $(p>0.1)$. Further analysis of patients of different ethnic origin again did not show significant differences in the allele frequencies.

Conclusion Major differences in the frequency of genetic polymorphisms in four candidate genes involved with FGF19 and bile acid metabolism are unlikely to be associated with primary BAD. However, the SNPs identified may contribute small effects, with a combination of SNPs in several genes producing a cumulative effect. Larger numbers of cases are required to define specific interactions or minor effects. At present the cause of the reduced FGF19 action in BAD remains uncertain.

Competing interests None.

Keywords Bile Acid Diarrhoea, Fibroblast Growth Factor 19, FXR, Klotho beta, OST alpha, single nucleotide polymorphisms. 